\title{
Results of liver transplantation in patients with acute liver failure due to Amanita phalloides and paracetamol (acetaminophen) intoxication
}

\author{
Maciej Krasnodębski, Michał Grąt, Wacław Hołówko, Łukasz Masior, Karolina M. Wronka, Karolina Grąt, \\ Jan Stypułkowski, Waldemar Patkowski, Marek Krawczyk
}

Department of General, Transplant, and Liver Surgery, Medical University of Warsaw, Warsaw, Poland

Gastroenterology Rev 2016; 11 (2): 90-95

DOI: $10.5114 / p g .2015 .52031$

Key words: acute liver failure, Amanita phalloides poisoning, paracetamol poisoning, liver transplantation.

Address for correspondence: Maciej Krasnodębski MD, Department of General, Transplant, and Liver Surgery, Medical University of Warsaw, 1 A Banacha St, 02-097 Warsaw, Poland, phone: +48 2259925 45, fax: +48 2259915 45, e-mail: mwkrasn@gmail.com

\begin{abstract}
Introduction: Amanita phalloides and paracetamol intoxications are responsible for the majority of acute liver failures. Aim: To assess survival outcomes and to analyse risk factors affecting survival in the studied group.

Material and methods: Of 1369 liver transplantations performed in the Department of General, Transplant, and Liver Surgery, Medical University of Warsaw before December 2013, 20 (1.46\%) patients with Amanita phalloides $(n=13,0.95 \%)$ and paracetamol $(n=7,0.51 \%)$ intoxication were selected for this retrospective study. Overall and graft survival at 5 years were set as primary outcome measures.

Results: Five-year overall survival after liver transplantation in the studied group was $53.57 \%$ and $53.85 \%$ in patients with paracetamol and Amanita phalloides poisoning, respectively $(p=0.816)$. Five-year graft survival was $26.79 \%$ for patients with paracetamol and $38.46 \%$ with Amanita phalloides intoxication $(p=0.737)$. Risk factors affecting patient survival were: pre-transplant bilirubin concentration $(p=0.023)$ and higher number of red blood cells $(p=0.013)$ and fresh frozen plasma $(p=0.004)$ transfused intraoperatively. Likewise, higher number of red blood cells $(p=0.012)$ and fresh frozen plasma $(p=0.007)$ transfused were risk factors affecting 5 -year graft survival. Surprisingly, donor and recipient blood type incompatibility was neither the risk factor for 5 -year overall survival $(p=0.939)$ nor the risk factor for 5 -year graft survival $(p=0.189)$.

Conclusions: In selected intoxicated patients urgent liver transplantation is the only successful modality of treatment. Risk factors affecting survival are in correspondence with the patient's pre-transplant status (bilirubin level in serum) and intraoperative status (number of red blood cells and fresh frozen plasma transfused).
\end{abstract}

\section{Introduction}

Acute liver failure is a condition in which all its functions cease to continue. There are many causes of this rare condition, with the most common being drug-induced hepatic injury (e.g. paracetamol-induced), infections with hepatitis A, B, or E viruses, Amanita phalloides intoxication, and narcotic use-related liver damage [1-4]. The existing O'Grady, Japanese, and Bernuau classifications divide acute liver failure based on the time period between jaundice and the onset of encephalopathy. In these classifications, terms such as "acute", "hyperacute", or "fulminant" liver failure are used [5-7]. The mortality of untreated patients reaches $90 \%$ [8] Initial treatment depends on the causes of hepatocel- lular injury and patients' general condition. In patients suspected of paracetamol-induced liver injury, prompt administration of $\mathrm{N}$-acetylcysteine (NAC) limits further hepatocellular injury, thus improving the patient's condition [9]. The choice of subsequent treatment has to be made as soon as acute liver failure is diagnosed. This decision may eventually lead to an urgent qualification for liver transplantation. Various criteria can be used in the decision-making process. The most commonly accepted and most widely used criteria are those presented by King's College authors [10]. However, their use in patients with acute paracetamol-induced liver failure is controversial [11]. Due to the usually severe condition of the patients on qualification and on-going doubts, it is vital that survival risk factors for both 
patient and transplanted organ (graft) are determined and examined.

\begin{abstract}
Aim
The aim of this study was to analyse the results of treatment and to determine risk factors affecting outcomes of patients with acute liver failure following paracetamol or Amanita phalloides poisoning, treated with urgent liver transplantation.
\end{abstract}

\section{Material and methods}

There have been 1369 liver transplantations carried out during the period between 1994 and 2013 in the Department of General, Transplant, and Liver Surgery (Medical University of Warsaw). For retrospective analysis, 20 patients (1.46\%) who underwent urgent liver transplantation due to Amanita phalloides ( $n=13$, $0.95 \%)$ or paracetamol $(n=7,0.51 \%)$ poisoning were chosen. The end points for this study were 5-year patient and graft survival rates.

\section{Statistical analysis}

Quantitative and qualitative variations are presented as medians (interquartile range) and numbers (\%). Mann-Whitney $U$ test and Fisher's exact test were used as appropriate. For survival calculations, the Kaplan-Meier method was used. The log-rank test was used for comparisons. Risk factors were determined based on Cox proportional hazards regression. Hazard ratios $(\mathrm{HR})$ are given with 95\% confidence intervals $(\mathrm{Cl})$. Statistica 10 (StatSoft Inc., Tulsa, USA) was used for analyses. The significance level was set at 0.05.

\section{Results}

Baseline characteristics of patients are presented in Table I. In the direct perioperative period 8 (40\%) patients died, and 1 (5\%) died during the surgery. Causes of death were as follows: multi-organ failure $(n=5)$, intraoperative bleeding $(n=1)$, septic shock $(n=1)$, and portal vein thrombosis $(n=1)$. Postoperative morbidity was $70 \%$ (14 of 20 ). The most frequent complications were: multi-organ failure (30\%) and postoperative hepatic artery thrombosis (15\%). Three patients required re-transplantation due to the following causes: hepatic artery thrombosis $(n=2)$ and acute graft rejection $(n=1)$. Five-year survival rates in patients with paracetamol and Amanita phalloides poisoning were $53.57 \%$ and $53.85 \%(p=0.816)$, respectively (Figure 1). Whereas the corresponding 5 -year graft survival rates were $26.79 \%$ and $38.46 \%$, respectively ( $p=0.737$, Figure 2 ).

Risk factors of overall survival included elevated preoperative serum bilirubin concentration $(p=0.023)$ and higher number of transfused blood units ( $p=0.013)$ and fresh frozen plasma ( $p=0.004)$ (Table II). Likewise, the number of transfused blood units $(p=0.012)$ and fresh frozen plasma ( $p=0.007)$ were significantly associated with graft survival (Table II). ABO-incompatible liver transplantations, however, did not prove to be a risk factor for either overall patient $(p=0.939)$ or graft ( $p=0.189$ ) survival.

\section{Discussion}

Intoxication caused by ingestion of mushrooms from Amanita phalloides species is responsible for the vast majority of mushroom poisonings. This rare cause of hepatotoxic injury results in mortality reaching $30 \%$ in all patients who ingest Amanita phalloides, and not only those who develope acute liver failure [12]. The toxicity of Amanita phalloides is related to two distinct groups of toxins: phallotoxins and amatoxins. Liver, renal, and enterocellular damage are caused mostly by amatoxins [13]. The onset of intoxication symptoms usually begins 6-12 $\mathrm{h}$ after ingestion and is divided into three consecutive phases - gastrointestinal, hepatotoxic, and the last one, with apparent hepato-renal syndrome, in the most severe poisoning, resulting in fulminant liver failure and death. To date, in spite of ongoing attempts and clinical trials, no effective medication has been discovered. In adjunctive therapy, penicillin G, and NAC are used, but with little effectiveness. In cases of fulminant liver failure, liver transplantation remains the only option (Figure 3).

A study from 2002, including 20-year data analysis from facilities in the United States and in Europe regarding Amanita phalloides poisonings, shows that only 32 out of 2108 hospitalised patients underwent liver transplantation [14]. Therefore, the number of patients in the Department of General, Transplant, and Liver Surgery at the Medical University of Warsaw seems even more interesting (it is a result of common harvesting and eating mushrooms in Poland, as well as the reference level of our facility regarding treatment with liver transplant). The small percentage of patients who underwent transplantation, and on the other hand, the high mortality reaching as much as $30 \%$, indicates that Amanita phalloides poisoning is a condition in which only collaboration with highly specialised facilities and making prompt decisions might save those patients.

Nevertheless, paracetamol intoxication is the most frequent cause of hepatotoxic damage [1] (Figure 3). Early NAC administration, i.e. up to $8 \mathrm{~h}$ from poisoning, results in significant reduction of hepatotoxicity risk and improvement of the patient's general condition [15]. In those whose condition was not improved after N-acetyl cysteine administration and implemented conservative 
Table I. Comparison of baseline characteristics between patients with paracetamol and Amanita phalloides intoxication

\begin{tabular}{|c|c|c|c|}
\hline Parameter & Paracetamol intoxication & Amanita phalloides intoxication & Value of $p$ \\
\hline Number of patients & 7 & 13 & - \\
\hline Sex (male) & $5(71.4 \%)$ & $6(46.2 \%)$ & 0.279 \\
\hline Age [years] & $21(19-26)$ & $48(45-50)$ & $<0.001$ \\
\hline Red blood cell count $\left[\mathrm{m} / \mathrm{n} / \mathrm{mm}^{3}\right]$ & $3.52(3.35-4.81)$ & $3.72(3.21-4.13)$ & 0.601 \\
\hline Haemoglobin value [g/dl] & $11.17(9.3-12.5)$ & $11.75(8.6-13.0)$ & 0.740 \\
\hline White blood cell count $\left[10^{3} / \mathrm{mm}^{3}\right]$ & $10.01(7.4-14.6)$ & $7(5.1-14.51)$ & 0.270 \\
\hline Platelet count $\left[10^{3} / \mathrm{mm}^{3}\right]$ & $122(45-156)$ & $75.5(44-119)$ & 0.417 \\
\hline Bilirubin value [mg/dl] & $5.4(4.12-8.4)$ & $5.82(3.62-7.97)$ & 0.860 \\
\hline Creatinine level [mg/dl] & $1.27(0.71-5.08)$ & $1.51(0.95-3.22)$ & 0.758 \\
\hline Albumin level [mg/ml] & $2.4(2.2-3.5)$ & $2.7(2.5-3.4)$ & 0.475 \\
\hline Blood urea level [mg/dl] & $60(17-90)$ & $40.31(21.2-97.7)$ & 1.000 \\
\hline AST [IU/I] & 2207 (95-5074) & $1134(68-1766)$ & 0.470 \\
\hline ALT [IU/I] & $2685(681-5581)$ & $950(309-3612)$ & 0.299 \\
\hline GGTP $[I U / I]$ & $100(48-402)$ & $78(53-223)$ & 0.669 \\
\hline ALP [IU/I] & $148(83-173)$ & $114(89-141)$ & 0.299 \\
\hline LDH [IU/I]) & $2309(695-12719)$ & $2106(1112-12161)$ & 0.710 \\
\hline INR & $2.27(2.06-5.13)$ & $3.43(2.77-4.89)$ & 0.417 \\
\hline MELD score & $31(21-40)$ & $29(27-34)$ & 0.962 \\
\hline Red blood cell transfusions & $4(3-4)$ & $6(5-8)$ & 0.060 \\
\hline Fresh frozen plasma transfusions & $9(8-10)$ & $9(7-14)$ & 1.000 \\
\hline Cold ischaemic time [h] & $8.5(7.8-9)$ & $9(7.3-9)$ & 0.837 \\
\hline Donor age [years] & $40(33-49)$ & $50(44-56)$ & 0.115 \\
\hline Blood group match & $6(85.71 \%)$ & $9(69.23 \%)$ & 0.417 \\
\hline
\end{tabular}

Qualitative and quantitative data are shown as the number (\%) and median (interquartile range), AST - aspartate transaminase, ALT - alanine transaminase, GGTP- $\gamma$-glutamyl transferase, ALP - alkaline phosphatase, LDH - lactate dehydrogenase, INR - international normalised ratio, MELD - Model of End-Stage Liver Disease.

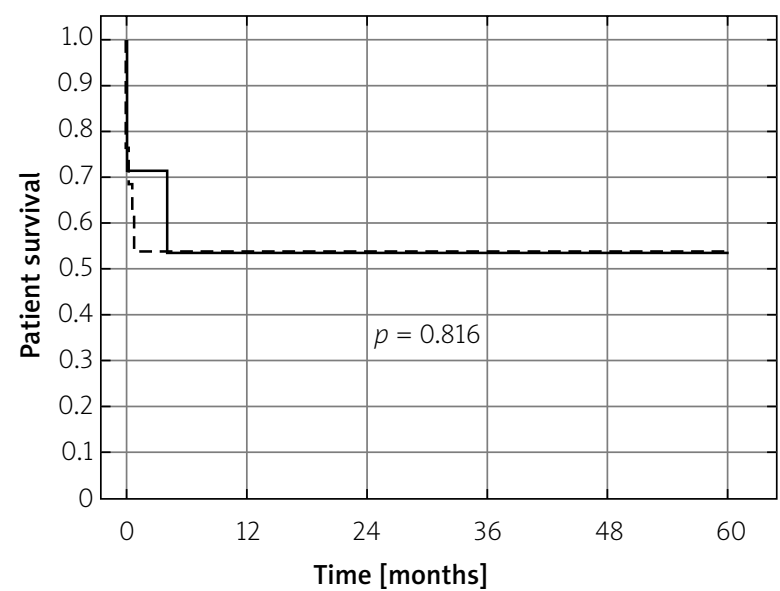

Figure 1. Five-year survival rates in patients with paracetamol (solid line) or Amanita phalloides (dotted line) treated with liver transplantation

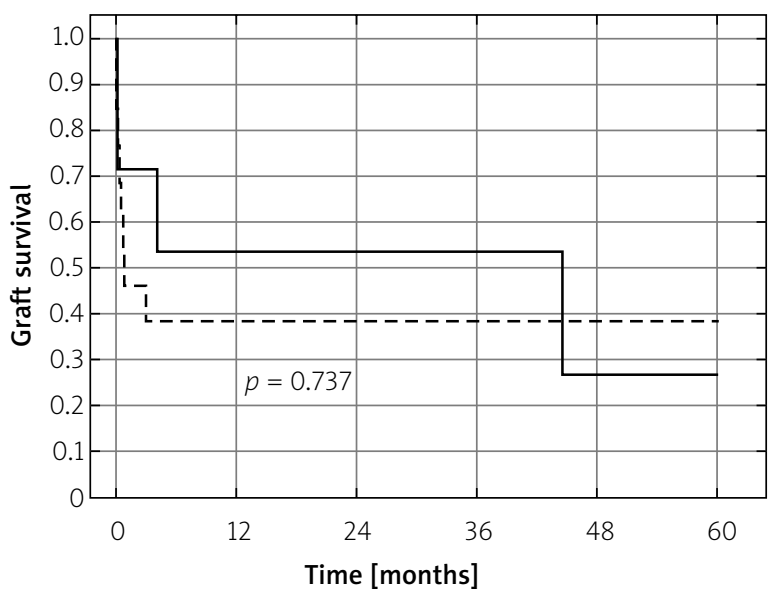

Figure 2. Five-year graft survival rates in patients with paracetamol (solid line) or Amanita phalloides (dotted line) 
Table II. Risk factors of overall survival and graft survival

\begin{tabular}{|c|c|c|c|c|}
\hline \multirow[t]{2}{*}{ Parameter } & \multicolumn{2}{|c|}{ Overall survival } & \multicolumn{2}{|c|}{ Graft survival } \\
\hline & $\mathrm{HR}(95 \% \mathrm{Cl})$ & Value of $p$ & $\mathrm{HR}(95 \% \mathrm{Cl})$ & Value of $p$ \\
\hline Amanita phalloides intoxication* & $1.171(0.293-4.689)$ & 0.823 & $1.217(0.365-4.050)$ & 0.749 \\
\hline Sex (male) & $1.594(0.422-6.026)$ & 0.491 & $1.401(0.443-4.425)$ & 0.566 \\
\hline Age [years] & $1.010(0.964-1.049)$ & 0.811 & $1.005(0.969-1.042)$ & 0.792 \\
\hline $\begin{array}{l}\text { Blood group mismatch between donor } \\
\text { and recipient }\end{array}$ & $0.940(0.195-4.535)$ & 0.939 & $2.294(0.665-7.914)$ & 0.189 \\
\hline Haemoglobin value [g/dl] & $0.841(0.652-13.0)$ & 0.184 & $0.901(0.704-1.521)$ & 0.406 \\
\hline Bilirubin value $[\mathrm{mg} / \mathrm{dl}]$ & $1.179(1.023-1.358)$ & 0.023 & $1.146(1.000-1.314)$ & 0.051 \\
\hline Creatinine level [mg/dl] & $1.161(0.958-1.407)$ & 0.127 & $1.120(0.931-1.348)$ & 0.229 \\
\hline AST [IU/I] & $0.998(0.970-1.027)$ & 0.896 & $0.994(0.965-1.023)$ & 0.676 \\
\hline $\mathrm{ALT}[\mathrm{IU} / \mathrm{I}]$ & $0.991(0.963-1.020)$ & 0.538 & $0.988(0.961-1.016)$ & 0.386 \\
\hline INR & $1.163(0.761-1.776)$ & 0.486 & $1.239(0.858-1.790)$ & 0.252 \\
\hline MELD score & $1.081(0.997-1.173)$ & 0.058 & $1.057(0.987-1.131)$ & 0.113 \\
\hline Red blood cell transfusions & $1.250(1.048-1.491)$ & 0.013 & $1.245(1.049-1.479)$ & 0.012 \\
\hline Fresh frozen plasma transfusions & $1.287(1.080-1.535)$ & 0.004 & $1.249(1.062-1.470)$ & 0.007 \\
\hline Cold ischaemic time [h] & $0.986(0.604-1.611)$ & 0.956 & $0.902(0.574-1.418)$ & 0.655 \\
\hline Donor age [ears] & 0.967 (0.909-1.029) & 0.296 & $0.988(0.936-1.044)$ & 0.672 \\
\hline
\end{tabular}

$H R$ - hazard ratios, $95 \% \mathrm{Cl}-95 \%$ confidence intervals, AST - aspartate transaminase, ALT - alanine transaminase, GGTP- $\gamma$-glutamyl transferase, ALP - alkaline phosphatase, $L D H$ - lactate dehydrogenase, INR - international normalized ratio, MELD - model of end-stage liver disease, *Vs. paracetamol intoxication. Hazard ratios for quantitative variables were given per: 1 year for donor and recipient age $1 \mathrm{~g} / \mathrm{dl}$ for haemoglobin concentration; per $1 \mathrm{mg} / \mathrm{dl}$ for serum bilirubin and creatinine concentrations; $1000 \mathrm{U} / \mathrm{l}$ for AST and ALT; 1 for INR; 1 point for MELD; 1 unit for transfusions; $1 \mathrm{~h}$ for cold ischaemic time.
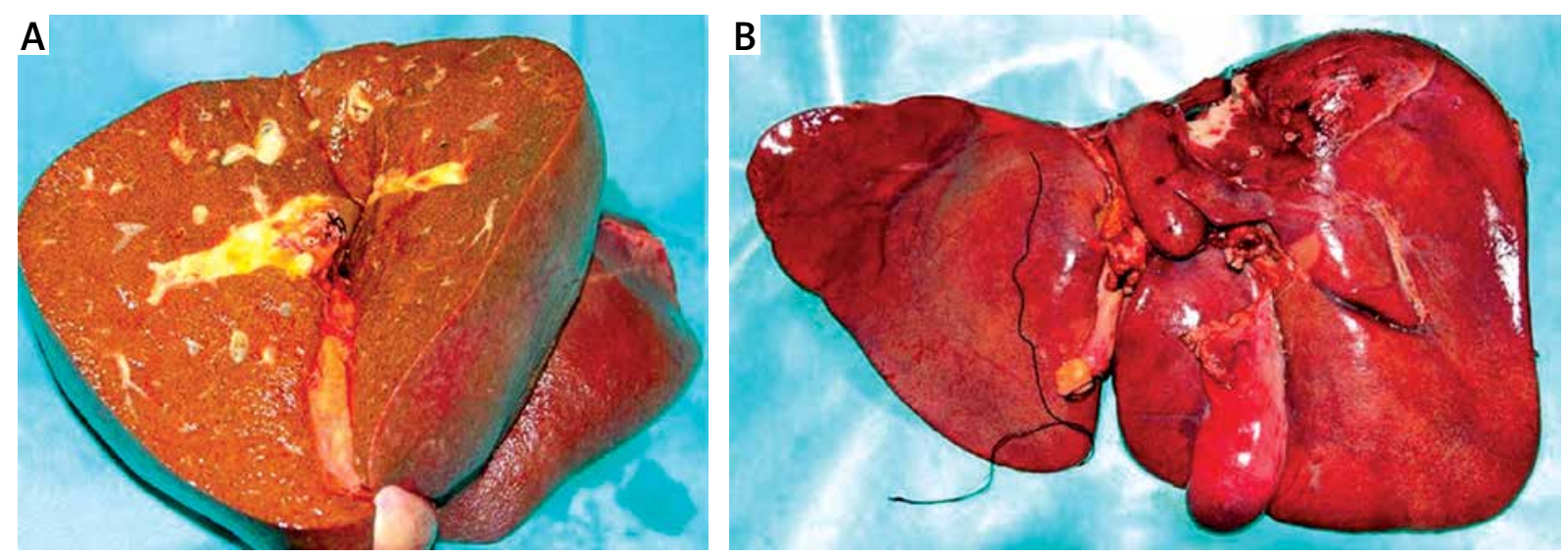

Figure 3. Liver damage due to Amanita phalloides intoxication (A) and paracetamol overdose (B)

therapy, liver transplantation remains the only treatment. Unlike Amanita phalloides poisoning, ingestion of which is usually accidental, paracetamol intoxication is often intentional (44\% of cases) [16], raising ethical concerns. One of the most important risk factors for successful suicidal attempt is a history of such in the past [17]. Thus, it is questionable if such patients should have equal access to liver transplants as do patients af- ter accidental poisoning [18]. The limited availability of organs accounts for a very thorough and just process of qualification for liver transplantation. The greatest problem and qualification limitation for urgent liver transplantation is clearly the passing of time. It is not always possible to determine the patient's intentions, especially when his/her condition deteriorates and even basic anamnesis is hard to obtain. Thus, it is crucial to access 
the patient thoroughly, regarding his/her medical condition and the environment around the patient after the transplantation, and, in cases where a suicide attempt is suspected, prompt psychiatric care should be provided.

It should be stressed that the results of liver transplantation in patients with acute liver failure, following either Amanita phalloides poisoning or paracetamol intoxication, are worse than those performed due to other indications [2, 3]. This comes from the fact that those patients are operated on in general poor condition, on an emergency basis. Failure does not only concern the liver, but often also the kidneys. Some patients develop cerebral oedema symptoms prior to transplantation. In analysed material, the paracetamol intoxication group included younger patients (median age 21 years). The young age of intoxicated patients is usually related to intentional actions and suicide attempts. Apart from that, no significant differences were found between groups. What is interesting, 5-year overall and graft survival rates were similar. Comparable survival rates in both groups suggest that the greatest risk for the patients is time period prior to transplantation. Their further survival, however, after a successful transplant, does not rely greatly on the cause of acute liver failure.

Higher serum bilirubin levels prior to transplantation turned out to be a negative risk factor for 5-year survival rates in these patients. This parameter is directly related to the condition and function of a damaged liver. In patients with acute liver failure following poisoning, not only is serum bilirubin elevated but also aminotransferase levels, and the prothrombin index decreases, cerebral oedema occurs, etc. Nevertheless, out of all biochemical tests only bilirubin elevation proved statistically significant as a poor prognosis factor. One way of minimising this effect might be either early qualification of a patient for transplantation (often the delay is related to late transfer of patients from other facilities) or prolonged waiting time for an organ. Obviously, the influence of the people involved in the treatment of these patients affects improvement only in the first of the aforementioned situations, i.e. patient's referral to high-reference facilities where medical doctors thoroughly assess and qualify patients for a possible transplantation. Notably, bilirubin level $>17.5 \mathrm{mg} / \mathrm{dl}$ is one of the King's College criteria for urgent liver transplantation due to indications other than paracetamol poisoning. This parameter alone should not be a reason for qualification, but in case of exceeding the abovementioned level it can be taken into consideration.

Other risk factors of poorer survival rates of both patients and grafts included larger number of transfused red blood cell units and fresh frozen plasma. Patients with acute liver failure present significant coagulopathy (INR reaching 7), which, from the beginning of the surgery, causes persistent bleeding requiring the use of volume expanders and haemostatic factors, such as recombinant coagulation factor VII a (NovoSeven). Nonetheless, bringing intraoperative bleeding under control is extremely difficult, if not impossible in some cases. These patients also present with a lack of portal hypertension, which results in a need for veno-venous bypass (bio-pump) and implementation of a surgical method involving excision of retrohepatic vena cava (classical surgical technique) (Figure 4). The amount of volume expanders transfused during the operation is influenced not only by the patient's general condition,
A

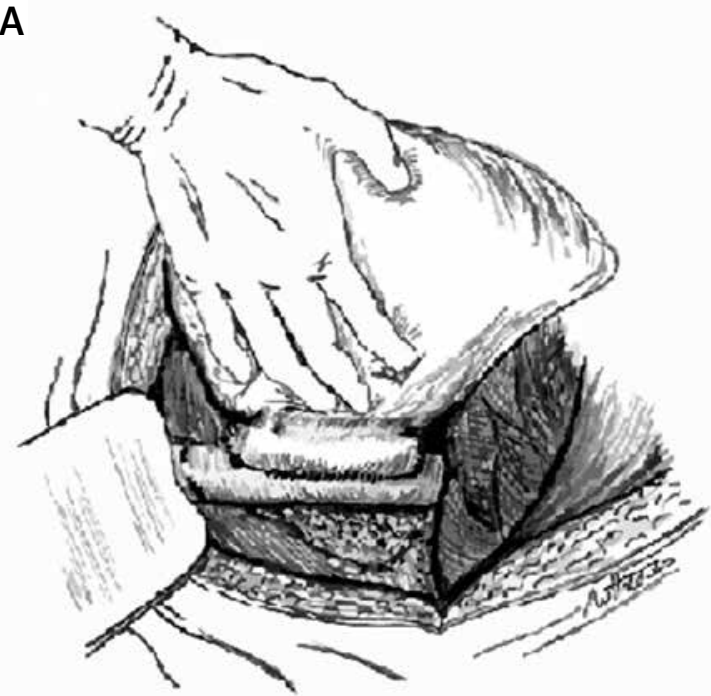

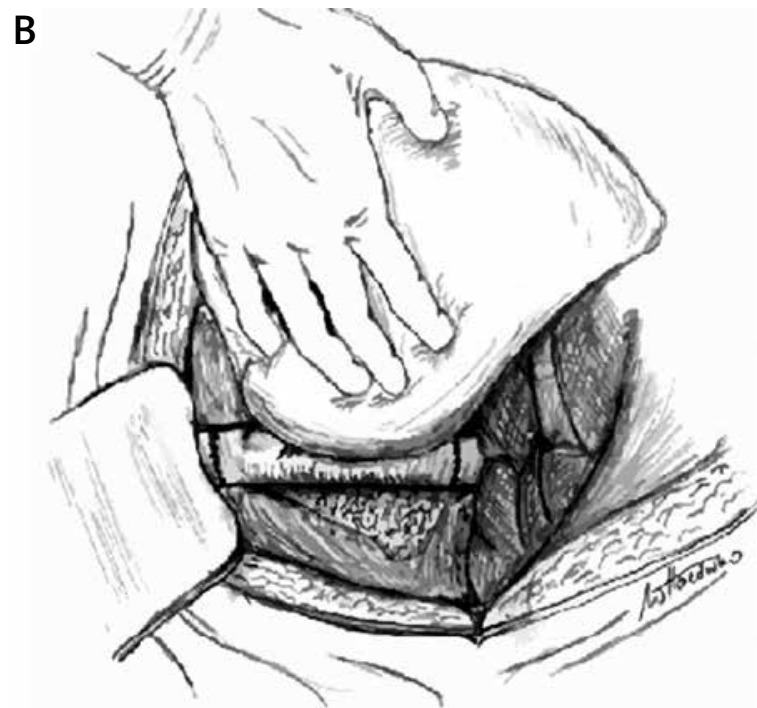

Figure 4. Side-to-side cavocavostomy technique (A) and classic technique for orthotopic liver transplantation (B) 
mainly being coagulopathy issues, but also technical conditions (surgeries in the past, if applicable, necessity of bio-pump use), and the duration and course of the surgery itself (classical surgical technique, operator's experience). For surgeons, the last aspect seems the most important one because it is the only one that depends on their actions, which affects further survival of the patient.

Paracetamol or Amanita phalloides poisoning are rare causes of liver transplantation. Patients whose condition deteriorates usually require urgent liver transplantation. It seems that the most crucial risk factor a surgeon has any influence on during the transplantation itself is the amount of transfused red blood cell units and fresh frozen plasma. This means that for this kind of surgery, experienced surgeons would be the most suitable operators. High levels of serum bilirubin prior to the surgery, being at the same time a survival risk factor, may indicate that some patients have been transferred to a reference centre too late.

\section{Conclusions}

Liver transplantation results are similar in patients with acute liver failure following Amanita phalloides and paracetamol intoxication. Risk factors affecting poor patient survival regard directly the patient's condition prior to transplantation, which is presented by serum bilirubin level. The second risk factor proved to be the amount of transfused packed red blood cell units and fresh frozen plasma.

\section{Conflict of interest}

The authors declare no conflict of interest.

\section{References}

1. Bernal W, Wendon J. Acute liver failure. N Engl J Med 2013; 369: 2525-34.

2. Krawczyk M, Grąt M, Barski K, et al. 1000 liver transplantations at the Department of General, Transplant and Liver Surgery, Medical University of Warsaw - analysis of indications and results. Pol Przegl Chir 2012; 84: 304-12.

3. Krawczyk M, Grat M, Kornasiewicz O, et al. Results of liver transplantation in the Department of General, Transplant and Liver Surgery at the Medical University of Warsaw in patients with chronic hepatitis B and C viruses infection. Przegl Epidemiol 2013; 67: 5-10, 93-7.

4. Nyckowski P, Skwarek A, Zieniewicz K, et al. Orthotopic liver transplantation for fulminant hepatic failure. Transplant Proc 2006; 38: 219-20.

5. O'Grady JG, Schalm SW, Williams R. Acute liver failure: redefining the syndromes. Lancet 1993; 342: 273-5.

6. Bernuau J, Rueff B, Benhamou JP. Fulminant and subfulminant liver failure: definitions and causes. Semin Liver Dis 1986; 6: 97-106.
7. Mochida S, Nakayama N, Matsui A, et al. Re-evaluation of the Guideline published by the Acute Liver Failure Study Group of Japan in 1996 to determine the indications of liver transplantation in patients with fulminant hepatitis. Hepatol Res 2008; 38: 970-9.

8. O'Grady JG. Acute liver failure. Postgrad Med J 2005; 81: 148-54.

9. Craig D, Bates C, Davidson J, et al. Staggered overdose pattern and delay to hospital presentation are associated with adverse outcomes following paracetamol induced hepatotoxicity. $\mathrm{Br}$ J Clin Pharmacol 2011; 73: 285-94.

10. O'Grady JG, Alexander GJ, Hayllar KM, et al. Early indicators of prognosis in fulminant hepatic failure. Gastroenterology 1989; 97: 439-45.

11. McPhail MJ, Wendon JA, Bernal W. Meta-analysis of performance of Kings's College Hospital Criteria in prediction of outcome in non-paracetamol-induced acute liver failure. J Hepatol 2010; 53: 492-9.

12. Escudie L, Francoz C, Vinel JP, et al. Amanita phalloides poisoning: assessment of prognostic factors and indications for emergency liver transplantation. J Hepatol 2007; 46: 466-73.

13. Vetter J. Toxins of Amanita phalloides. Toxicon 1998; 36: 13-24.

14. Enjalbert F, Rapior S, Nouguier-Soulé J, et al. Treatment of amatoxin poisoning: 20-year retrospective analysis. Toxicol Clin Toxicol 2002; 40: 715-57.

15. Ortolani O, Conti A, de Gaudio AR, et al. The effect of glutathione and $\mathrm{N}$-acetylcysteine on lipoperoxidative damage in patients with early septic shock. Am J Respir Crit Care Med 2000; 161: 1907-11.

16. Larson AM, Polson J, Fontana RJ, et al. Acetaminophen-induced acute liver failure: results of a United States multicenter, prospective study. Hepatology 2005; 42: 1364-72.

17. Nordentoft M, Breum L, Munck LK, et al. High mortality by natural and unnatural causes: a 10 year follow up study of patients admitted to a poisoning treatment centre after suicide attempts. BMJ 1993; 306: 1637-41.

18. Rhodes R, Aggarwal S, Schiano TD, et al. Overdose with suicidal intent: ethical considerations for liver transplant programs. Liver Transplantation 2011; 17: 1111-6.

Received: 27.01.2015

Accepted: 17.03 .2015 